water vapour, is facilitated during periods of photosynthesis, and water loss is curtailed at night. But stomata also close sometimes during the day where the air is dry and excessive evaporation places the plant under water stress.

It is now well established that light causes stomata to open by lowering the carbon dioxide concentration in the vicinity of the guard cells as a result of photosynthesis. By this device increased consumption of carbon dioxide by the leaf leads to stimulation of absorption and vice versa. The response to changes in the humidity of the air has been less intensively investigated, but it has been generally accepted that it depends on alterations in the water content of the leaf as a whole. Lange, of the Botanical Institute, University of Würzburg, has dissociated himself from this view and in a recent article in Planta $(100,76 ; 1971)$ he and his colleagues put forward further evidence that guard cells respond directly to changes in the humidity gradient existing between the external air and substomatal air space.

Lange et al. have devised an elegant technique whereby it is possible to observe microscopically the behaviour of stomata in isolated strips of leaf epidermis when subjected to rapid changes of ambient air of different moisture contents. Induction of opening and closing by alternate exposure of the outer surface of the epidermis to moist and dry air was repeated as many as fifteen times on the same stoma. When different areas of the same piece of epidermis were treated separately the stomata in each part responded independently according to the conditions to which they were subjected.

Lange et al. conclude that guard cells act as humidity sensors which respond directly to changes in the gradient of water potential between the air above the leaf and in the sub-stomatal air space. When this gradient increases the guard cells lose water and the pore closes and when it falls they take up water causing the pore to open. This mechanism causes a rapid adjustment in pore size in response to changes in evaporative conditions in the vicinity of each stoma irrespective of the water status of the leaf as a whole. Lange et al. assume that the level of carbon dioxide was unaffected by the treatments that were applied and that the changes in turgidity of the guard cells is attributable to a direct influence of the humidity gradient on absorption and loss of water by these cells. The possibility of an interaction between carbon dioxide concentration and water potential gradient in the overall control of stomatal response will no doubt be examined in the future extension of this work.

\title{
Satellite Lines in the Solar X-Ray Spectrum
}

IN next Monday's Nature Physical Science, J. H. Parkinson of the University of Leicester reports observations of satellite lines in the solar X-ray spectrum. These are lines which are observed close to the resonance transition of each helium-like ion, hence the name "satellite".

During the past few years the study of helium-like ion spectra and associated satellite lines has proved to be of considerable interest to solar physicists. In addition to the resonance line, a partially forbidden "intercombination" line is observed for each ion. A further strong line at slightly longer wavelength than the resonance and intercombination lines was shown by Gabriel and Jordan (Nature, 221, 947; $1969)$ to originate from a transition which was previously thought to be highly forbidden as a one quantum transition. These authors (Mon. Not. Roy. Astron. Soc., 145, 241; 1969) then showed that the relative intensity of the intercombination and forbidden lines can depend on the electron density of the emitting plasma.

The satellite lines are caused by transitions in the stage of ionization lower than the helium-like ions, that is, in the lithium-like ions. The resonance line of the helium-like ion is a $1 \mathrm{~s}^{2}-1 \mathrm{~s} 2 \mathrm{p}$ transition. The satellite lines have a similar wavelength to this transition because they are transitions of the same type but in the presence of a further electron (that is, $1 \mathrm{~s}^{2} \mathrm{n} l-1 \mathrm{~s} 2 \mathrm{p} \mathrm{n} l$ ). Satellite lines have been observed in laboratory spectra for many years-Edlén and Tyrén first reported them in 1939, and proposed the forementioned origin.

As part of the investigation leading to the identification of the helium-like ion forbidden transition, Gabriel and Jordan classified several multiplets of satellite lines, because it was at one time suspected that the strong solar line could have a similar origin. They proposed that the excited levels giving rise to the satellite lines are populated by the process of di-electronic recombination, hitherto not reported as a process important in laboratory plasmas, but thought to be important at the lower densities in the sola corona.

Several groups of workers (at the Aerospace Corporation, the Goddard Space Flight Center, the Lebedev Institute, and the US Naval Research Laboratory) have recently reported the presence of weak satellite lines in solar spectra, chiefly during flare activity. The new observations by Parkinson were obtained with improved spatial resolution by using a collimated crystal spectrometer, and refer to a stable phase of the active region. Parkinson's intensity data indicate that, as in the laboratory, the solar satellite lines are formed by the process of di-electronic recombination; this demonstrates directly the existence of this process in the solar corona. The relative intensities of the satellite lines and helium-like resonance lines are therefore dependent on the electron temperature in the emitting plasma. Parkinson has observed the intensities of the helium-like lines and satellite lines, so both the electron density and temperature in the emitting solar active region can be studied. And because each group of lines occurs over a limited wavelength region it should be possible to obtain reliable relative intensities.

\section{Aspirin and Human Embryonic Cells in Culture}

IN next Wednesday's Nature New Biology, Paine and Nagington report that sodium salicylate - a breakdown product of the common sodium acetylsalicylate or aspirin-inhibits the growth of some human embryonic cells in culture.

Tissue cultures from skin, lung, heart and kidney were prepared from 12,14 and 18-week old human embryos. The cells were grown in the presence of various concentrations of sodium salicylate. Kidney cells from 12 week old embryos showed the highest sensitivity $-2 \mathrm{mM}(320 \mu \mathrm{g} / \mathrm{ml}$.) salicylate completely inhibited growth and $0.5 \mathrm{mM}$ $(80 \mu \mathrm{g} / \mathrm{ml}$.) almost reduced the growth rate by half. Lung cells were also affected, but less markedly. Heart cells were even less sensitive than kidney and lung cells; only $2 \mathrm{mM}(320 \mu \mathrm{g} / \mathrm{ml}$.) salicylate showed any significant effect at all. Skin fibroblasts were not inhibited, even by $2 \mathrm{mM}$ salicylate.
The mode by which salicylates produce this inhibition is unknown. Salicylates have a wide variety of pharmacological effects. There are indications of a correlation between salicylates taken during early pregnảncy and foetal defects; but a causal connexion has not been established. Analysis of the foetal blood of 272 consecutive deliveries showed that about 10 per cent had salicylate concentrations of more than $10 \mu \mathrm{g} / \mathrm{ml}$; t the mean concentration in these infants was $33 \mu \mathrm{g} / \mathrm{ml}$. (about $0.2 \mathrm{mM}$ ) (Palmisano and Cassady, J. Amer. Med. Assoc., 209, 556 ; 1969).

Paine and Nagington quite properly point out that one cannot infer that the action of a drug in an intact animal or human is similar to the effect of the drug on cells in tissue culture. But the evidence they present is enough to warrant further investigations. 